\title{
Morphing Mechanisms Part 2: Using Repetitive Control to Morph Cam Follower Motion
}

\author{
${ }^{1}$ Nonglak Phetkong, ${ }^{2}$ Meng-Sang Chew and ${ }^{3}$ Richard W. Longman \\ ${ }^{1}$ The National Metal and Materials Technology, Center (MTEC), Patumthani, Thailand 12120 \\ ${ }^{2}$ Dempartment of Mechanical Engineering, Lehigh University, Bethlehem, PA 18015 USA \\ ${ }^{3}$ Department of Mechanical Engineering, Columbia University \\ New York, New York 10027 USA
}

\begin{abstract}
This research utilizes the repetitive control approach in morphing a high-speed cam mechanism so that its output displacement tracks the desired output trajectory. The main objective is to reduce the output tracking error, which is the difference between the actual and desired output displacements of the output mass. A 3-degree of freedom mathematical model is used to analyze and represent the cam follower system. The output tracking error in cam follower systems is generally caused by errors in cam profile manufacturing, variation in the speed of the driving motor and the dynamics of the cam follower system. Theoretical and experimental results show that the output tracking error is greatly reduced after repetitive control has been applied to the system. As a result, the actual output displacement tracks the desired output displacement very closely.
\end{abstract}

Key words: Modern Control, Nonlinear Cam Follower, Tracking Error Reduction, Vibration in Cam Mechanisms

\section{INTRODUCTION}

This study seeks to reduce the tracking error in the output displacement of a high-speed cam follower system theoretically and experimentally by using the repetitive control method. The work presented here is a continuation of the work by Phetkong et al. ${ }^{[1]}$, which has utilized the learning control theory to morph cam mechanisms. Learning control is an off-line process. The system stops after the end of each cycle in order to calculate the next input. Thus, the learning approach may not be suitable for any processes which are continuous. Repetitive control, therefore, plays significant roles in continuous (real-time) processes. Similar to the learning control algorithm, repetitive control modifies its input for the next cycle based on the tracking error (the difference between the actual and desired outputs) and the input from the previous cycle. In addition, repetitive control also takes into account the difference in the initial conditions of the current and previous cycles in order to keep the system running continuously.

In this study, repetitive control is applied continuously to the cam follower system in order to morph the output of a 2-3 polynomial cam into a 3-4-5 polynomial desired trajectory.

Previous Investigations: Dynamic analyses of cam mechanisms have been of interest among many researchers for decades. A brief list of researchers involved in this topic during the past few decades includes Chew et al. ${ }^{[2,}$ 3] who investigated the application of optimal control theory to the synthesis of high-speed cam-follower systems in 1983. In 1989, Phan et al. ${ }^{[4]}$ investigated indirect repetitive control theory for linear discrete multivariable systems. Chew and $\mathrm{Phan}^{[5,6]}$, used learning control theory in reducing residual vibrations in electromechanical high-speed cam follower systems. Learning control is an off-line process and is a sub-category of repetitive control, which is a continuous process. In 1996, Chang ${ }^{[7]}$ applied repetitive control theory to a one degree-offreedom cam-follower system in order to reduce residual vibrations in the system.

Repetitive Control Theory: Repetitive control is an extension of the learning control theory. It is more suitable for continuous processes which require an online algorithm modification. The process continues from one cycle to the next to the actual output tracks the desired output trajectory within a specified tolerance. Repetitive control equations and procedures are given by Phan et al. ${ }^{[4]}$ and are mentioned here briefly.

The continuous-time state space equation of the cam-follower system is given by the following equation:

$$
\dot{\boldsymbol{x}}(t)=\boldsymbol{A}_{c} \boldsymbol{x}(t)+\boldsymbol{B}_{\mathrm{c}} \boldsymbol{u}(t)
$$

The output equation is given as follows:

$Y(t)=C x(t)$ 
The next step is the estimation of system parameters. In repetitive control, a controller is designed for an estimated system, given by:

$\boldsymbol{Y}_{\mathrm{j}}=\boldsymbol{A}_{\mathrm{j}} \boldsymbol{x}_{\mathrm{j}}(0)+\boldsymbol{B}_{\mathrm{j}} \boldsymbol{u}_{\mathrm{j}}$

Where, $j$ is the cycle number:

$\boldsymbol{Y}_{\mathrm{j}}=\left[\begin{array}{c}\mathrm{y}_{\mathrm{j}}(\Delta \mathrm{t}) \\ \mathrm{y}_{\mathrm{j}}(2 \Delta \mathrm{t}) \\ \ldots \\ \ldots \\ \mathrm{y}_{\mathrm{j}}(\mathrm{p})\end{array}\right], \boldsymbol{u}_{\mathrm{j}}=\left[\begin{array}{c}\mathrm{u}_{\mathrm{j}}(0) \\ \mathrm{u}_{\mathrm{j}}(\Delta \mathrm{t}) \\ \ldots \\ \ldots \\ \mathrm{u}_{\mathrm{j}}(\mathrm{p}-\Delta \mathrm{t})\end{array}\right]$,

and $p$ is the period.

$\boldsymbol{A}$ and $\boldsymbol{B}$ is unknowns which will be obtained through the repetitive control process.

Equation (3) can be written in the form:

$\boldsymbol{Y}_{\mathrm{j}}=\boldsymbol{\Phi}_{\mathrm{j}} \boldsymbol{\Psi}_{\mathrm{j}}$,

Where, $\boldsymbol{\Phi}_{\mathrm{j}}=\left[\begin{array}{ll}\boldsymbol{A}_{\mathrm{j}} & \boldsymbol{B}_{\mathrm{j}}\end{array}\right]$ and

$$
\boldsymbol{\Psi}_{\mathrm{j}}=\left[\begin{array}{c}
\underline{x}_{j}^{\mathrm{T}}(0) \\
\mathrm{u}_{\mathrm{j}}(0) \\
\mathrm{u}_{\mathrm{j}}(\Delta t) \\
\mathrm{u}_{\mathrm{j}}(2 \Delta \mathrm{t}) \\
\ldots \\
\mathrm{u}_{\mathrm{j}}(\mathrm{p}-\Delta \mathrm{t})
\end{array}\right] .
$$
follows:

The repetitive control procedure is described as

Step 1: During the initial cycle (cycle \#0), an initial input, $\boldsymbol{u}_{0}$, is applied to the system. The corresponding output, $\boldsymbol{Y}_{0}$, is measured and recorded. Next, the initial conditions for cycle $\# 1, \boldsymbol{x}_{l}(0)$, are obtained from ending values of $\boldsymbol{Y}_{0}$. The input and output vectors are given below:

$\underline{\mathrm{u}}_{0}=\left[\begin{array}{c}\mathrm{u}(0) \\ \mathrm{u}(\Delta \mathrm{t}) \\ \ldots \\ \ldots \\ \mathrm{u}(\mathrm{p}-\Delta \mathrm{t})\end{array}\right], \underline{\mathrm{Y}}_{0}=\left[\begin{array}{c}\mathrm{y}(\Delta \mathrm{t}) \\ \mathrm{y}(2 \Delta \mathrm{t}) \\ \ldots \\ \ldots \\ \mathrm{y}(\mathrm{p})\end{array}\right]$

Step 2: The initial guesses for $\underline{A}_{0}$ and $\underline{B}_{0}$ are constructed and then the input for cycle \#1 is calculated based on the following equation.

$\boldsymbol{u}_{\mathrm{j}}=\boldsymbol{u}_{\mathrm{j}-1}+\boldsymbol{G}_{\mathrm{j}}\left[\boldsymbol{Y} *-\boldsymbol{Y}_{\mathrm{j}-1}-\boldsymbol{A}_{\mathrm{j}-1}\left\{\boldsymbol{x}_{\mathrm{j}}(0)-\boldsymbol{x}_{\mathrm{j}-1}(0)\right\}\right]$
Where, $\boldsymbol{G}_{\mathrm{j}}=\left(\boldsymbol{B}_{j}^{\mathrm{T}} \boldsymbol{Q} \boldsymbol{B}_{j}+\boldsymbol{S}\right)^{-1} \boldsymbol{B}_{j}^{\mathrm{T}} \boldsymbol{Q}$

Step 3: During cycle \#1, $\boldsymbol{u}_{l}$ is applied to the system and the corresponding output $\left(\boldsymbol{Y}_{l}\right)$ is measured. The initial conditions for cycle \#2, $\boldsymbol{x}_{2}(0)$, are obtained from ending values of $\boldsymbol{Y}_{l}$.

Step 4: $\boldsymbol{A}_{0}$ and $\boldsymbol{B}_{0}$ are updated to $\boldsymbol{A}_{l}$ and $\boldsymbol{B}_{l}$ according to Eqn. (4) and the following equation:

$\boldsymbol{\Phi}_{\mathrm{j}}=\boldsymbol{\Phi}_{\mathrm{j}-1}+\frac{\left(\boldsymbol{Y}_{\mathrm{j}}-\boldsymbol{\Phi}_{\mathrm{j}-1} \boldsymbol{\Psi}_{\mathrm{j}}\right) \boldsymbol{\Psi}_{\mathrm{j}}^{\mathrm{T}} \boldsymbol{R}_{\mathrm{j}-1}}{1+\boldsymbol{\Psi}_{\mathrm{j}}^{\mathrm{T}} \boldsymbol{R}_{\mathrm{j}-1} \boldsymbol{\Psi}_{\mathrm{j}}}$

$\boldsymbol{R}_{j}$ is called the projection matrix, with $\boldsymbol{R}_{0}=\alpha \underline{\boldsymbol{I}}_{(q x q)}, \alpha>$ 0 and $q=p+n$, where $n$ is the number of the state variables, $\boldsymbol{x}(t) . \boldsymbol{R}_{j}$ is updated as follows:

$$
\boldsymbol{R}_{\mathrm{j}}=\boldsymbol{R}_{\mathrm{j}-1}-\frac{\boldsymbol{R}_{\mathrm{j}-1} \boldsymbol{\Psi}_{\mathrm{j}} \boldsymbol{\Psi}_{\mathrm{j}}^{\mathrm{T}} \boldsymbol{R}_{\mathrm{j}-1}}{1+\boldsymbol{\Psi}_{\mathrm{j}}^{\mathrm{T}} \boldsymbol{R}_{\mathrm{j}} \boldsymbol{\Psi}_{\mathrm{j}}} .
$$

Step 5: The input for the next cycle, $u_{j}$, is calculated based on Eqn. (5) and the procedure continues.

The procedure and parameter updates are better explained with the aid of Fig. 1.

\section{THEORETICAL RESULTS}

The overall system's mathematical model is shown in Fig. 2. The input to the system is the voltage and the output of the system is the displacement of the output mass. The cam follower system has three degrees of freedom (3-DOF). Torsion and bending in the camshaft produce the first two degrees of freedom and the third degree of freedom is from the displacement of the output mass. Parameters used in the theoretical simulation and in the experiment are shown in Table 1.

Figure 3 shows the actual output of the cam follower system during the initial cycle when repetitive control has not been applied to the system. The actual output deviates significantly from the desired output. Next, repetitive control is applied to the system starting from cycle \#1. The first five continuous outputs are shown in Fig. 4. The output of the final cycle (cycle \#20), shown in Fig. 5, has converged closely to the desired output and can be seen by the error comparison in Fig. 6. The initial and final voltage inputs to the motor are shown in Fig. 7. It is evident that repetitive control has modified the voltage input to the motor so that the actual output converges to the desired output as the number of cycles increases. The initial and final errors are presented in Fig. 8. The final error has been reduced by approximately $95 \%$ of its initial value. 
Table 1: Repetitive Control Parameters Used in the Theoretical Simulation

Simulation:

Period

1 second

Number of intervals

50

The initial estimated parameters:

$$
\begin{aligned}
& \boldsymbol{A}_{\text {rise }}=\boldsymbol{Z e r o s}_{15 \times 8} \\
& \boldsymbol{B}_{\text {rise }}=\boldsymbol{I}_{15 \times 15} \\
& \boldsymbol{Q}_{\text {rise }}=\boldsymbol{I}_{15 \times 15} \\
& \boldsymbol{S}_{\text {rise }}=1.5 \times 10^{-8} \boldsymbol{I}_{15 \times 15} \\
& \boldsymbol{R}_{\text {rise }}=1.0 \times 10^{40} \boldsymbol{I}_{23 \times 23} \\
& \boldsymbol{A}_{\text {return }}=\boldsymbol{Z e r o s}_{15 \times 8} \\
& \boldsymbol{B}_{\text {return }}=\boldsymbol{I}_{15 \times 15} \\
& \boldsymbol{Q}_{\text {return }}=\boldsymbol{I}_{15 \times 15} \\
& \boldsymbol{S}_{\text {return }}=3.0 \times 10^{-7} \boldsymbol{I}_{15 \times 15} \\
& \boldsymbol{R}_{\text {return }}=1.0 \times 10^{30} \boldsymbol{I}_{23 \times 23}
\end{aligned}
$$

Experiment:

Period

2 seconds

Number of intervals

60

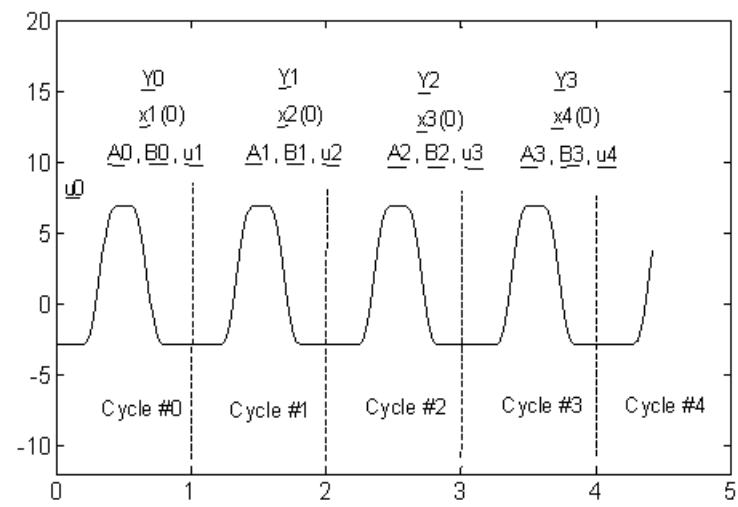

Fig. 1: Repetitive Control Procedure

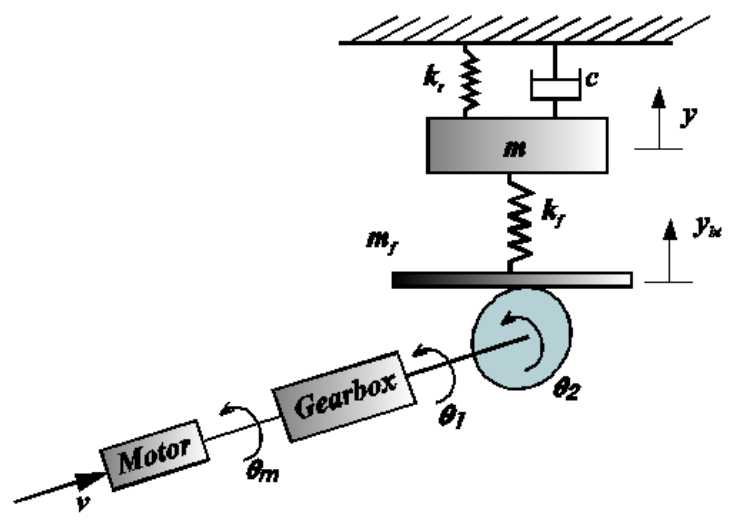

Fig. 2: An Overall Electromechanical System with a Motor

Experimental Setup: The overall view of the experimental setup is shown in Fig. 9.

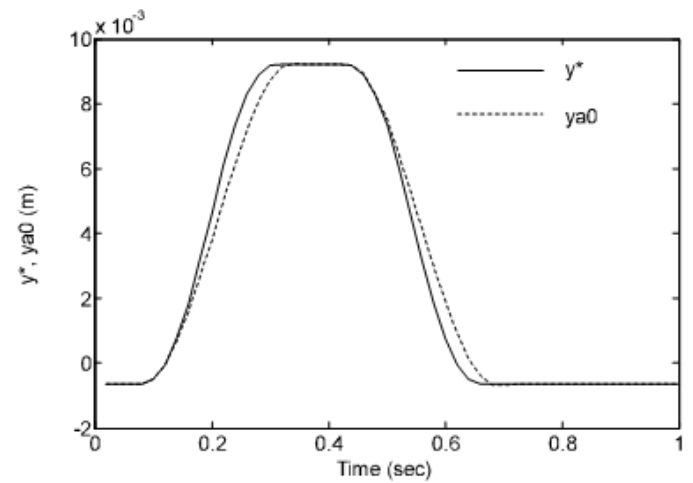

Fig. 3: Desired Output $\left(y^{*}\right)$ and Actual Output from the Initial Cycle

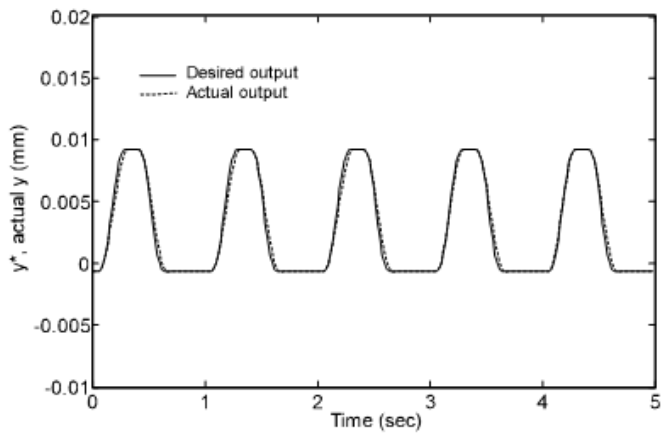

Fig. 4: Continuous Output of the First Five Cycles Compared to the Desired Output

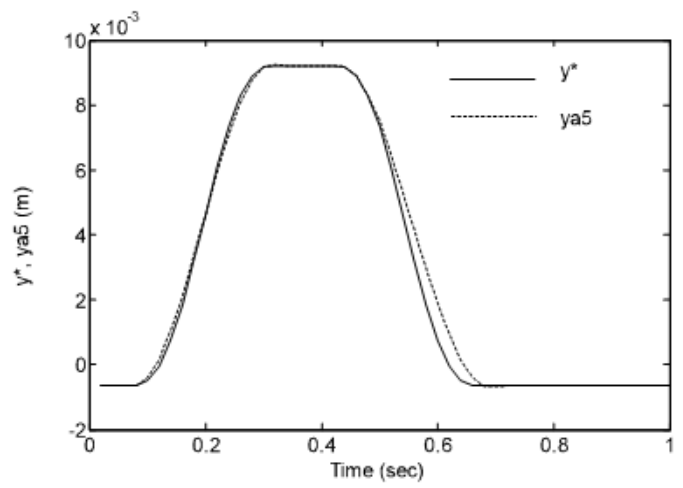

Fig. 5: Desired Output $\left(\mathrm{y}^{*}\right)$ and Actual Output from Cycle \#5

The experimental system consists of a cam-follower system, a DC motor, an encoder, a laser diode, a Position Sensing Detector (PSD) and amplifier, a servo voltage amplifier and a host personal computer. A d SPACE (Digital Signal Processing and Control Engineering) control hardware package is used for data acquisition and control purposes. The d SPACE package consists of a floating-point processor board, a multi-I/O board and an incremental encoder board. These boards are add-ons and are installed in the computer. 


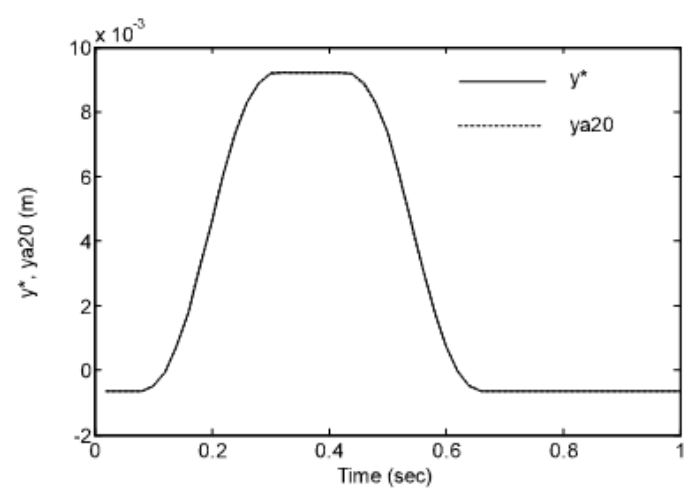

Fig. 6: Desired Output $\left(\mathrm{y}^{*}\right)$ and Actual Output from the Final Cycle

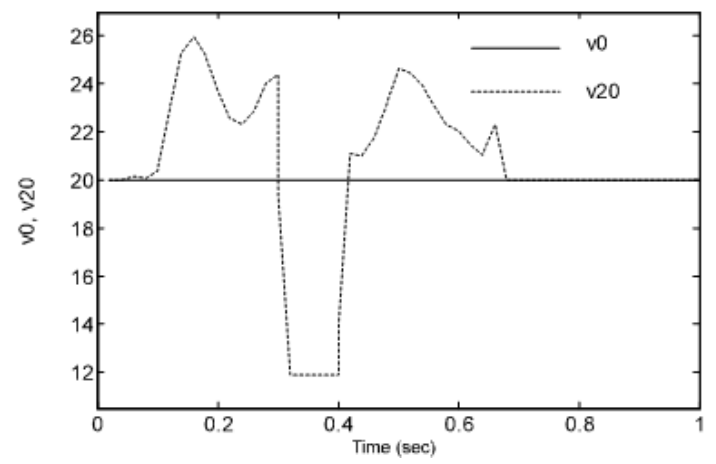

Fig. 7: Initial $\left(\mathrm{u}_{0}\right)$ and Final Input

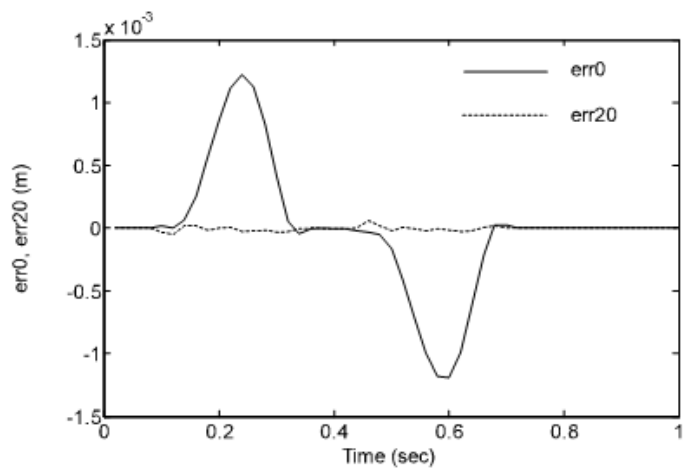

Fig. 8: Initial and Final Tracking Errors

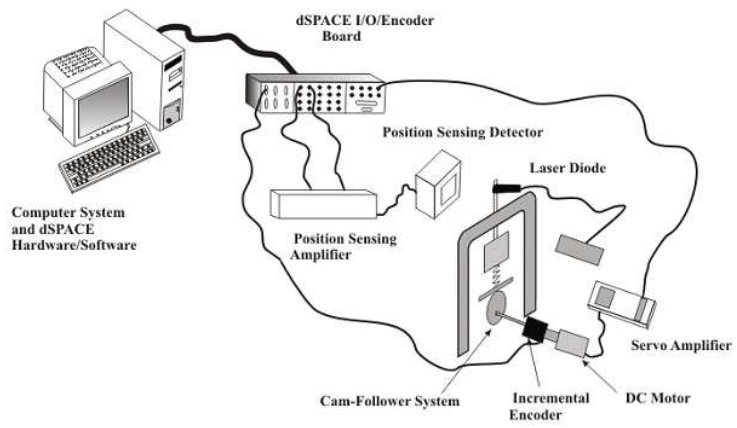

Fig. 9: Overall View of the Experimental Setup

\section{EXPERIMENTAL RESULTS}

Continuous outputs from the first five cycles are shown in Fig. 10. During the initial cycle, the system is operated without the control algorithm. The corresponding actual output displacement of the system, shown in Fig. 11, significantly deviates from the desired output trajectory. Repetitive control is applied to the first bottom dwellers, rise segment, top dwell and return segment, respectively, from cycle \#1 onward. Figure 12 shows the actual and desired trajectories from cycle \#3 which is the end of the learning process of the rise segment.

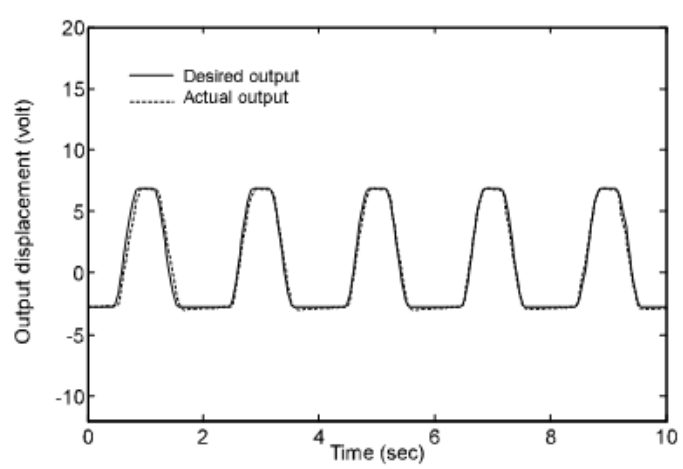

Fig. 10: Continuous Output of the First Five Cycles

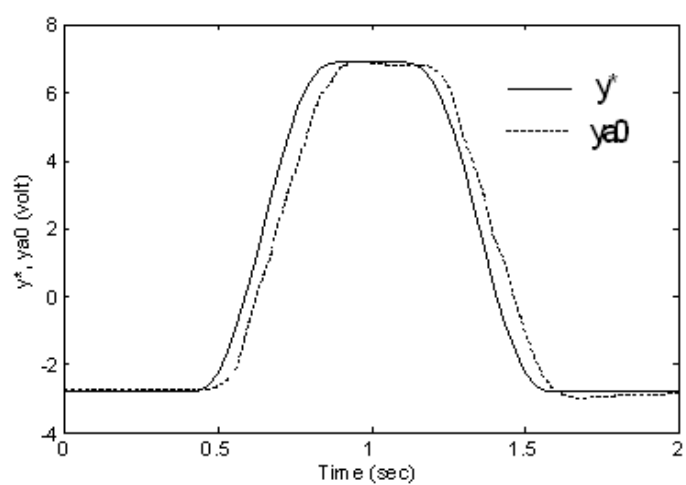

Fig. 11: Desired Output $\left(\mathrm{y}^{*}\right)$ and Actual Output from the Initial Cycle

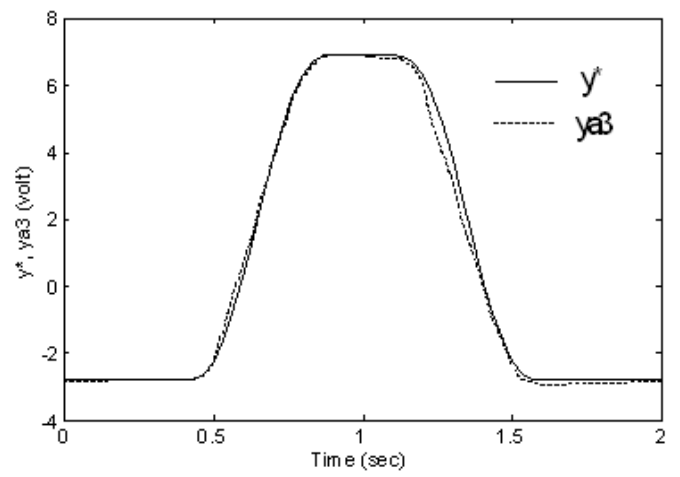

Fig. 12: Desired Output $\left(\mathrm{y}^{*}\right)$ and Actual Output from Cycle \#3 


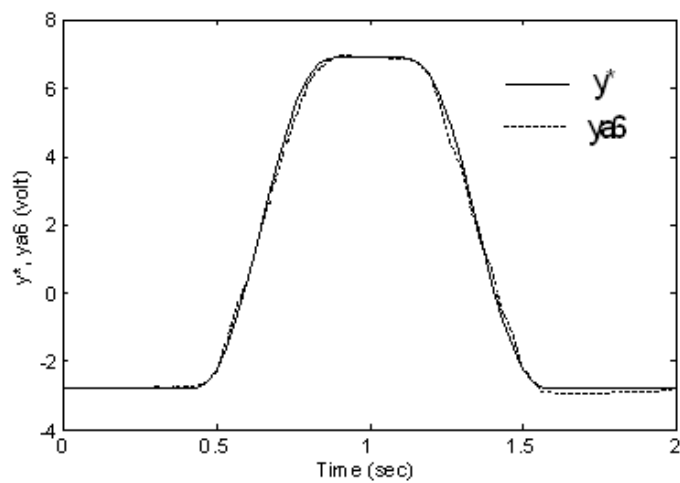

Fig. 13: Desired Output $\left(\mathrm{y}^{*}\right)$ and Actual Output from Cycle \#6

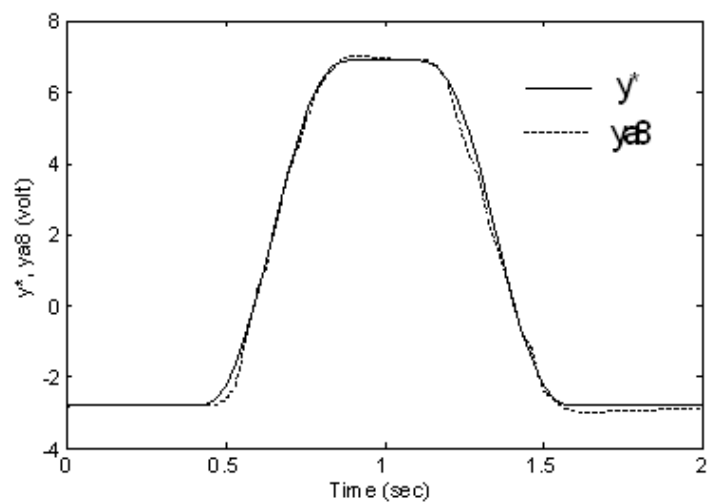

Fig. 14: Desired Output $\left(y^{*}\right)$ and Actual Output from Cycle \#8

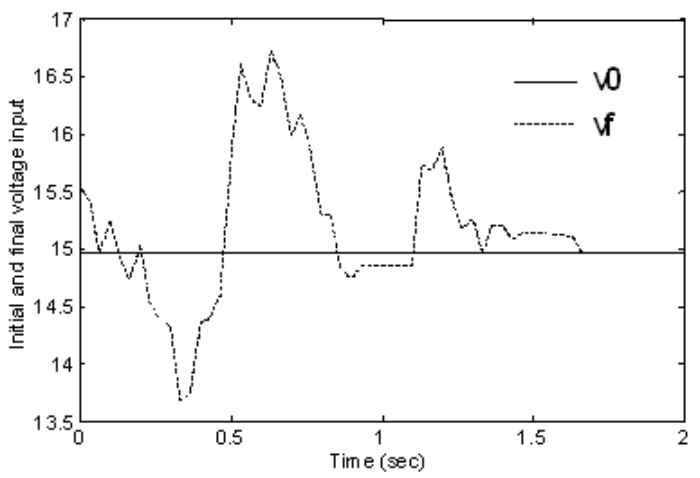

Fig. 15: Initial and Final Voltage Input

The results from the learning of the top dwell are shown in Fig. 13 where the actual and desired return segments are now starting at the same time. Results from the final cycle are shown in Fig. 14. We have shown that repetitive control has modified the voltage input of the system so that the actual output closely tracks the desired output. The initial and final voltage inputs to the motor are shown in Fig. 15.

Figure 16 shows deviations of the actual cam profile (dashed line) from the ideal profile (solid line).

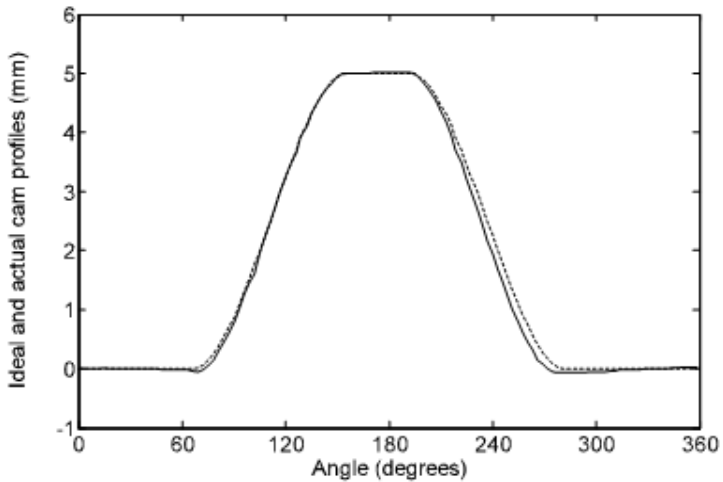

Fig. 16: Ideal and Actual Cam Profiles

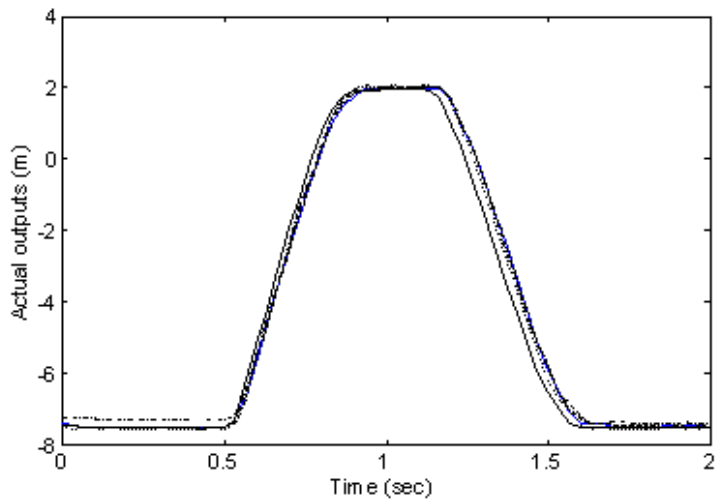

Fig. 17: System Repeatability Check

The ideal profile is the intended profile, but in fabricating the cam the actual profile still contained some manufacturing errors even with the use of a high precision NC machine.

The system repeatability, which is the ability of the system to generate the same output when subject to the same input, is shown in Fig. 17. The same input was sent to the system several times and their corresponding outputs were measured and compared. In theory, these outputs should be exactly the same. However, this is not the case in the experiment. A low level of system repeatability error was built into the system. Therefore, this error also exists in the experimental results. This is the main reason why the experimental output displacement does not track the desired output as exactly as in theory.

\section{CONCLUSION}

This study utilized the repetitive control algorithm theoretically and experimentally and has shown that the tracking error of a 3-DOF high-speed cam follower has been reduced significantly. The repetitive control theory does not require any prior knowledge of the parameters used in the system. Instead, it treats the system as a black box and modifies the new input to the system in order to reduce the tracking error. It is an excellent and intelligent tool to compensate for the 
errors in the cam profile, dynamic effects as well as variation in motor speed and other errors that cause the actual output displacement to deviate from the desired trajectory.

One crucial limitation of the experiment is the system repeatability. This is the ability of the system to produce the same output when subject to the same input. As shown earlier, the repeating error played an important role in the experimental results. Regardless of the repeating error, we have shown that the experimental output displacement tracks the desired output displacement to an acceptable accuracy.

\section{ACKNOWLEDGMENTS}

The authors would like to thank Lehigh University, Columbia University and the National Metal and Materials Technology Center of Thailand (MTEC) for their support.

Appendix: The 2-3 polynomial cam profile is described by the following equation:

$\mathrm{y}_{\mathrm{c}}(\theta)=\mathrm{H}_{\mathrm{c}}\left(3 \xi^{2}-2 \xi^{3}\right)$

Where, $\xi=\frac{\theta}{\beta_{1}}, \beta_{1}$ is the rise angle and $H_{c}$ is the cam lift.

The desired output displacement, which is a 3-4-5 polynomial, is described by:

$\mathrm{y}^{*}=\mathrm{H}\left(10 \xi^{3}-15 \xi^{4}+6 \xi^{5}\right)$

Where, $\xi=\frac{\theta}{\beta_{1}}$ and $\mathrm{H}$ is the maximum lift of the output mass and is equal to $\left(\frac{\mathrm{k}_{\mathrm{f}}}{\mathrm{k}_{\mathrm{f}}+\mathrm{k}_{\mathrm{r}}}\right) \mathrm{H}_{\mathrm{c}}$.

\section{REFERENCES}

1. Phetkong, N., M. Chew and R.W. Longman, 2005. Morphing Mechanisms Part 2: Using Repetitive Control to Morph Cam Follower Motion. American J. Appl. Sci., 2: 1.

2. Chew, M., F. Freudenstein and R.W. Longman, 1983. Application of optimal control theory to the synthesis of high-speed cam follower systems. Part 1: Optimality criterion. J. of Mechanisms, Transmissions and Automation in Design, Trans. Of ASME, 105: 576-584.

3. Chew, M., F. Freudenstein and R.W. Longman, 1983. Application of optimal control theory to the synthesis of high-speed cam follower systems. Part 2: System optimization. J. Of Mechanisms, Transmissions and Automation Design, Trans. Of ASME, 105: 585-591.

4. Phan, M., R.W. Longman and J. Juang, 1989. Indirect repetitive control of linear discrete multivariable systems. Proceedings of the $27^{\text {th }}$ Annual Allerton Conference on Communication, Control and Computing, Monticello, IL, pp: 867876.

5. Chew, M. and M. Phan, 1994. Application of learning control theory to mechanisms part 1: inverse kinematics and parametric error compensation. Machine Elements and Machine Dynamics, 71: 25-32.

6. Chew, M. and M. Phan, 1994. Application of learning control theory to mechanisms part 2: reduction of residual vibrations in high-speed electromechanical bonding machines. Machine Elements and Machine Dynamics, 71: 33-39.

7. Chang, W., 1996. Repetitive control of a highspeed cam-follower system. M.S. Thesis, Lehigh University, Bethlehem, PA, USA. 